\title{
Multiscale Characterization of Strain-Hardening Cementitious Composites
}

\author{
John E. Bolander ${ }^{1}$, Jingu Kang ${ }^{1}$, Yaming Pan $^{1}$ \\ ${ }^{1}$ Civil and Environmental Engineering, University of California, Davis, CA 95616 USA
}

\begin{abstract}
This research involves the multiscale characterization of strain-hardening cementitious composites under tensile loading. The sensitivity of cracking behavior to fiber dispersion is studied using three-dimensional lattice models, in which each fiber is explicitly represented. It is shown that the nonlocal modeling of fiber bridging forces is essential for obtaining realistic patterns of crack development. Crack count and crack size are simulated for progressively larger levels of tensile strain. The influence of fiber dispersion is clearly evident: regions with significantly fewer fibers act as defects, reducing strength and strain capacity of the material.
\end{abstract}

Keywords: strain-hardening, fracture, fiber debonding, lattice modeling, multiscale characterization

\section{Introduction}

Short dispersed fibers are commonly added to concrete materials to prevent or reduce crack openings. With appropriate constituents and design, such materials strain-harden and exhibit numerous fine cracks, rather than few large ones [1]. These strain-hardening cementitious composites (SHCC) are being developed for many applications, including those that require high toughness or long-term durability. It is understood that crack openings depend on local variations in the fiber content: regions with fewer fibers act as defects within the material and promote larger crack openings [2]. However, quantitative approaches that relate fiber dispersion (and other aspects of material design) to cracking behavior are few. To address this need, the authors have developed multiscale models of SHCC, in which individual fibers are explicitly represented within a lattice representation of the cement-based matrix. This paper describes recent improvements in the micromechanical modeling of fibers within these SHCC models. In particular, the bridging forces of individual fibers are distributed along their embedded lengths, which provides more realistic simulations of multiple cracking. Correlations between crack count, crack width, and characteristics of the fiber distribution are evident.

\section{Lattice modeling of fiber reinforced cement composites}

Elasticity and fracture of the matrix phase are represented by a lattice model, which is based on the rigid-bodyspring concept [3]. Fibers are introduced into the material domain irrespective of the discretization of the matrix phase. The representation of fibers is computationally efficient, making it applicable to a variety of problems where fiber distribution is important [4]. Prior to matrix cracking, the stiffness contributions of fibers are represented using elastic shear lag theory. After cracking, fiber contributions to strength and stiffness can be modeled using either: 1) a lumped-force approach [3], in which the fiber bridging force is lumped at matrix nodes $i$ and $j$ adjacent to the crack surface (Fig. 1a); or 2) a distributed-force approach [5], in which the bridging force and associated moments are distributed to the nodes along each embedded length of the fiber (Fig. 1b). Whereas fibers are assumed to be straight herein, the analysis framework can accommodate curved fibers or hooked-end fibers, provided the effects of the fiber geometries on the pullout force distribution are known.

Presenting and corresponding author: John E. Bolander, Civil and Environmental Engineering, University of California, Davis, CA 95616 USA; E-mail: jebolander@ucdavis.edu 
(a)

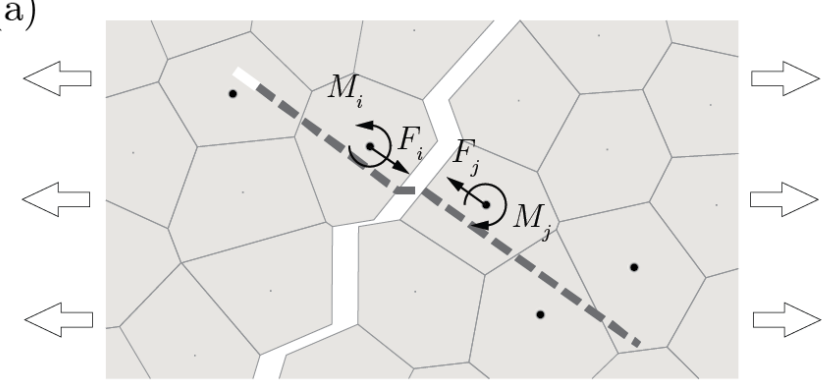

(b)

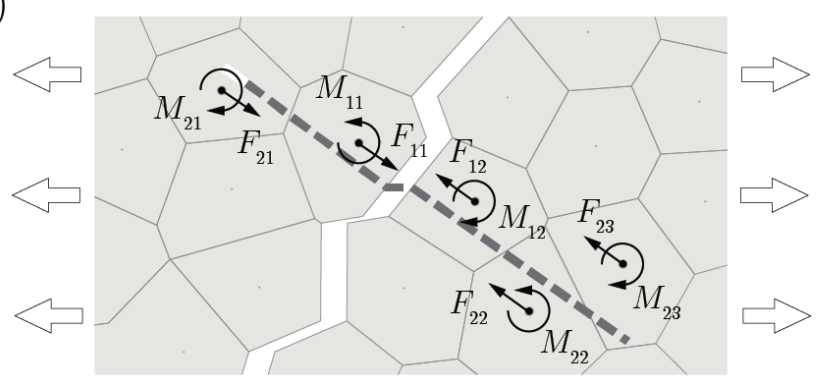

Figure 1: Lattice models of fiber bridging of a matrix tensile crack: (a) lumped-force approach; (b) distributed-force approach [5]

(a)

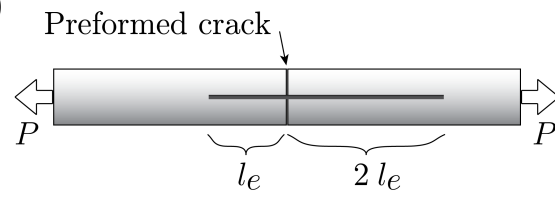

(b)

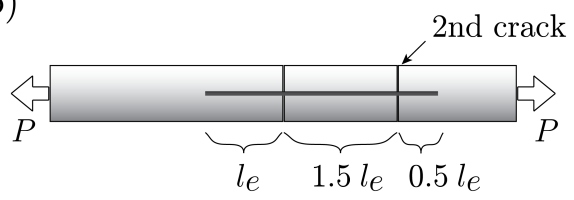

(c)

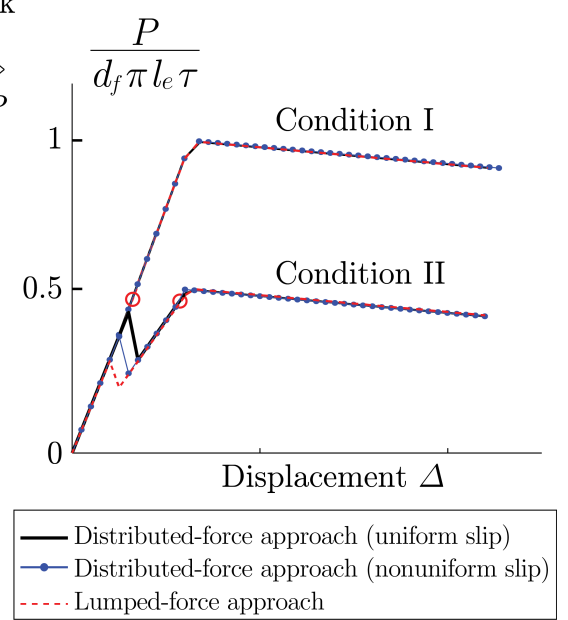

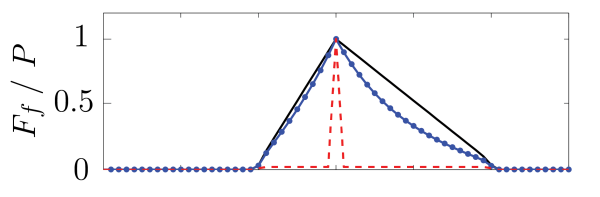

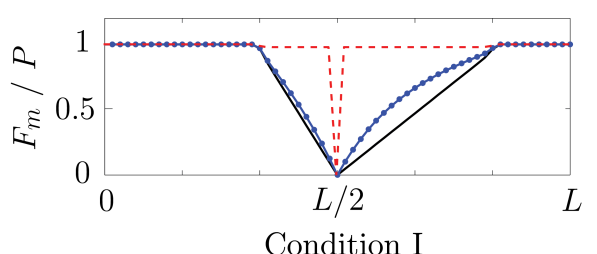

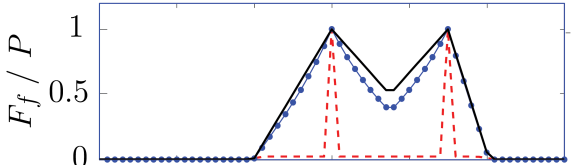

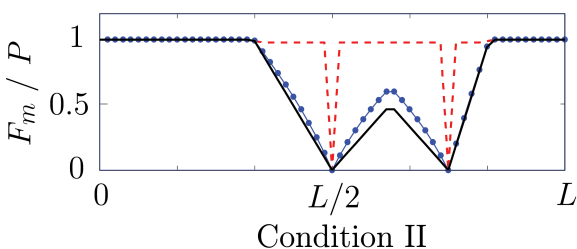

Lumped-force approach

Figure 2: Simulated fiber bridging forces, $F_{f}$, and matrix forces, $F_{m}$, under external load $P$ : (a) condition I: preformed matrix crack; (b) condition II: preformed crack plus additional matrix crack; (c) corresponding load-displacement responses

Simulated bridging forces are presented in Fig. 2 for a single fiber within a tensile specimen that has been discretized using 100 block-like lattice elements, as explained later in this paper. For condition I, only the preformed crack is assumed to be active (i.e., additional cracking is not permitted); for condition II, a weak zone has been introduced to induce the second crack. Along with the lumped-force approach, two formulations of the distributedforce approach are compared: uniform slip and nonuniform slip along the fiber-matrix interface according to the micromechanical model of Naaman et al. [6]. The lumped-force approach exhibits an unrealistically abrupt transfer of load from the fiber to the matrix, which can lead to spurious cracking on the adjacent matrix elements, particularly for $3 \mathrm{D}$ discretizations of the matrix [5]. In contrast, for both formulations of the distributed-force approach, the bridging force is transferred over the embedded lengths of the fiber. The nonuniform slip formulation provides a more realistic representation of progressive debonding of the fiber-matrix interface. Figure 2c shows the load-displacement curves for each cracking condition, where $d_{f}$ is the fiber diameter and $\tau$ is the interfacial bond strength. Peak load is lower for condition II due to the shorter embedment length of $0.5 l_{e}$. The load at which the second crack forms depends on the force carried by the matrix, such that the lowest cracking load occurs for the lumped-force model.

\section{Effects of fiber dispersion on SHCC cracking behavior}

\subsection{Discretization of tensile specimen}

The prismatic region of a tensile specimen is discretized using a block-like lattice model, as shown in Fig. 3 . Short fibers are placed within the central $100 \mathrm{~mm}$ length of the domain to achieve a volume fraction $V_{f}=2.0 \%$. Fiber centroids and orientations are determined semi-randomly [3]. For the distribution shown in Fig. 3a, the probability of fiber placement in the horizontal direction is conditioned on a sine function. Each of the 63,000 fibers is explicitly represented within the material domain. The fibers are $0.04 \mathrm{~mm}$ in diameter and $12 \mathrm{~mm}$ in length; the fiber/matrix 
(a)

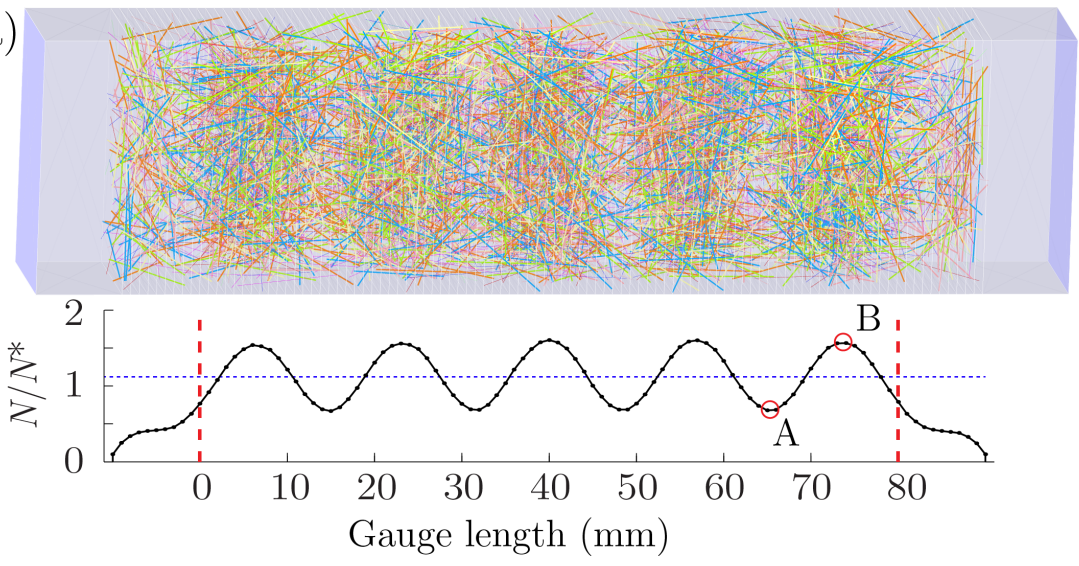

(b)

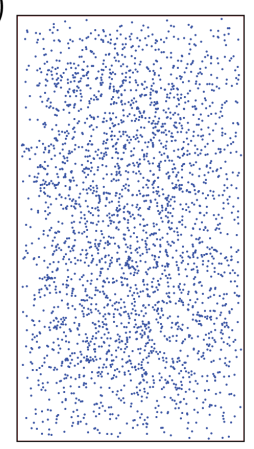

Section A

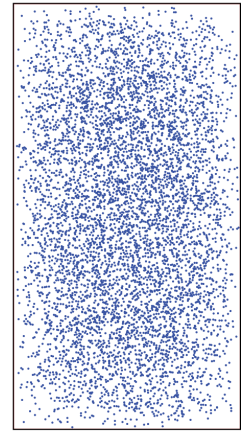

Section B

Figure 3: Discretized tensile specimen: (a) fiber distribution and domain discretization; (b) fiber intersections at cross-sections A and B

modular ratio is approximately 2. In Fig. 3a, $N$ is the number of fibers intersecting a cross-section (with dimensions of $30 \times 16 \mathrm{~mm}^{2}$ ) and $N^{*}$ is the theoretical number of intersections for a uniformly random 3D fiber distribution (i.e., $N^{*}=0.5 V_{f} A_{c} / A_{f}$, where $A_{f}$ and $A_{c}$ are the fiber and composite sectional areas, respectively).

\subsection{Analysis results}

To analyze the influence of fiber dispersion on cracking behavior, uniform and nonuniform fiber distributions are considered. The matrix is assumed to have uniform strength. The distributed-force approach (uniform slip formulation) is used for the analyses. Figure 4 presents analysis results for $1 \%$ tensile strain, $\varepsilon_{g}$, measured over the gauge length.

(a)
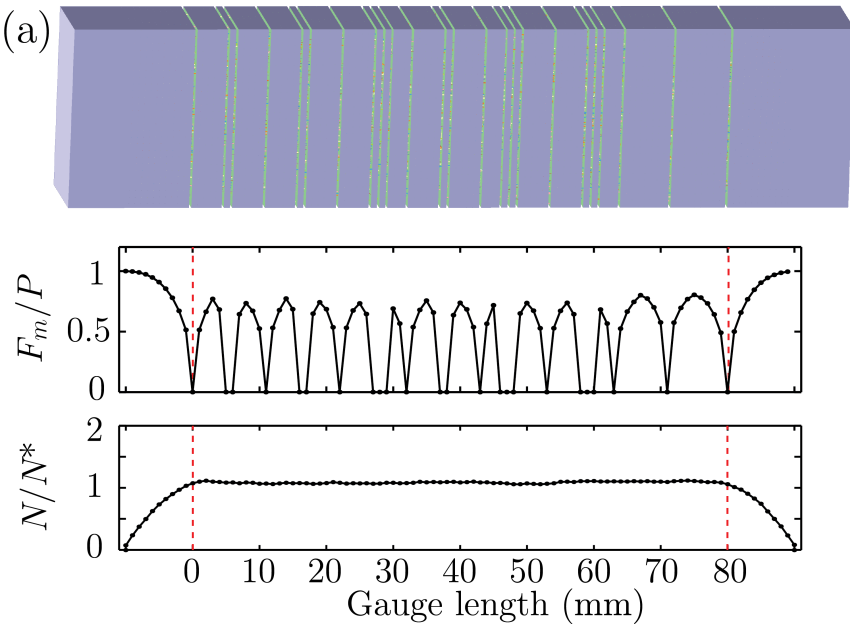

(b)
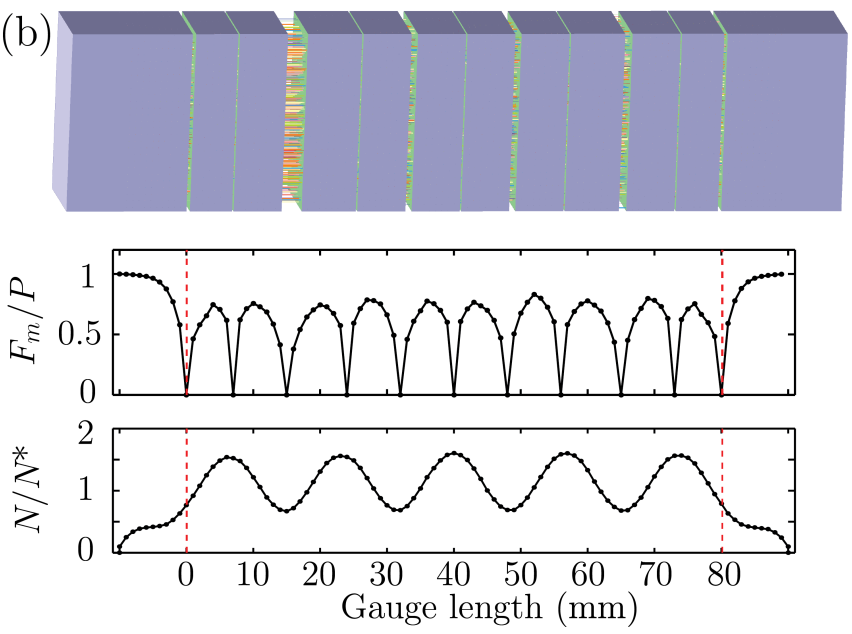

Figure 4: Crack patterns and matrix forces at 1\% strain using uniform slip model: (a) uniform fiber dispersion; (b) nonuniform fiber dispersion. Larger cracks appear at locations of lower fiber content

In the case of uniform fiber dispersion (Fig. 4a), a relatively larger number of irregularly spaced cracks is observed. In contrast, nonuniform fiber dispersions exhibit fewer cracks, with crack widths being larger near the troughs of the distribution profile. Figure 5a shows crack width histograms for several strain levels over the gauge length. As expected, the number of cracks increases with increasing levels of strain. For the case of uniform fiber dispersion, however, the crack widths are nearly constant at $0.04 \mathrm{~mm}$. In contrast, the nonuniform fiber distribution leads to more variation in crack widths and earlier crack localization and thus reduced stain capacity (Fig. 5b). In agreement with other studies [7], uniform dispersion leads to more a ductile composite. 

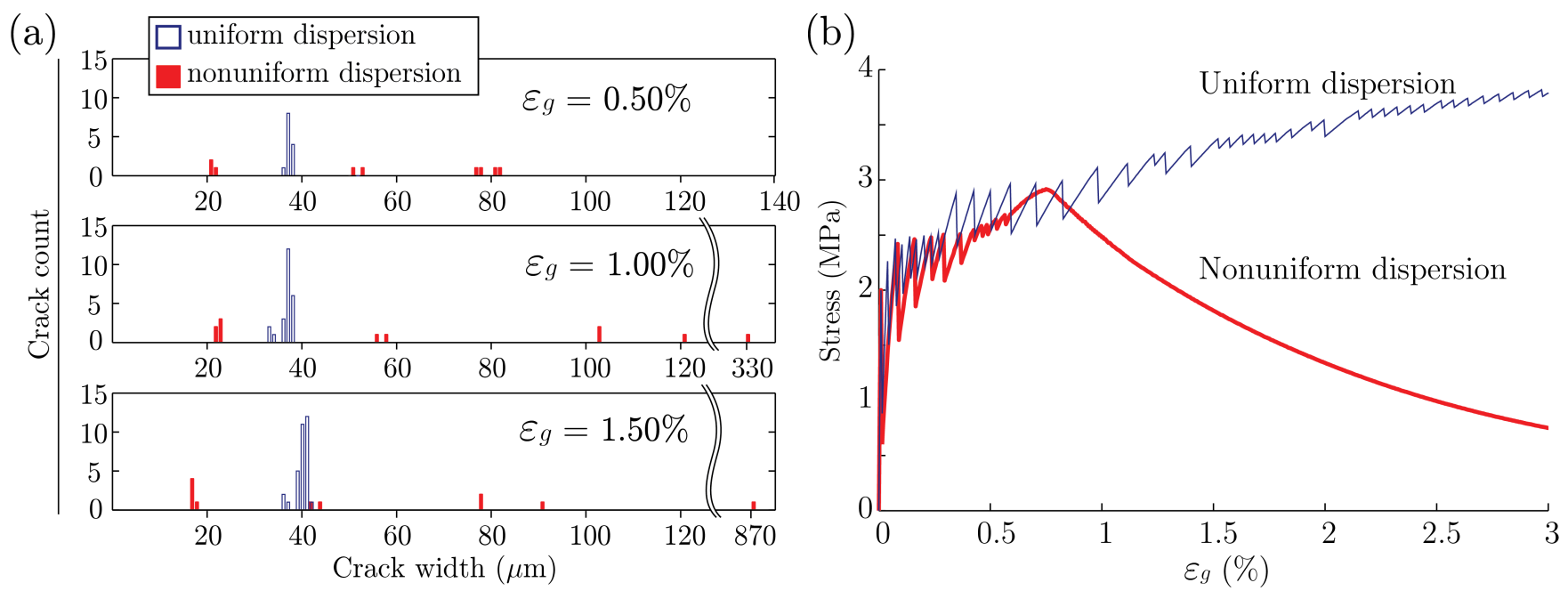

Figure 5: Influences of fiber dispersion and strain level on crack count and crack width: (a) crack-width histograms; (b) stress-strain responses

\section{Conclusions}

This paper reports on recent developments of multiscale models of strain-hardening cement composites, in which individual fibers are explicitly represented within a lattice model of the matrix phase. The multiscale character of the model is evident in the quantitative links established between the micromechanics of the fiber-matrix interface and composite performance measures, such as toughness and cracking behavior. In particular, this form of model is an effective means for studying the effects of fiber dispersion on cracking behavior. The following observations and conclusions are made.

1. The proposed distributed-force (nonlocal) approaches realistically simulate the transfer of cracking bridging forces over the fiber embedment lengths and associated effects on cracking patterns. The nonuniform slip formulation provides more accurate representations of the process of fiber debonding and the transfer of forces to the matrix. However, the assumption of uniform slip along the fiber length is simpler and gives comparably accurate representations of crack opening.

2. As expected, crack opening is correlated to the local volume fraction of fibers. Cracking occurs earlier and cracks are larger at locations with smaller fiber contents.

3. Uniformly random fiber dispersions lead to the development of distributed fine cracks of similar size and large strain capacity of the composite. On the other hand, significantly nonuniform fiber dispersions reduce ultimate strength and strain capacity.

4. Ongoing work involves comparisons with physical test results. For this purpose, realistic fiber dispersions are being constructed from image analyses of cross-sections produced by the serial sectioning of test specimens.

\section{References}

1. A.E. Naaman and H.W. Reinhardt (eds.), High Performance Fiber Reinforced Cement Composites 2 (HPFRCC 2): E \& FN SPON, London, 1996, $502 \mathrm{pp}$.

2. Y. Akkaya, S.P. Shah, and B. Ankenman, Effect of fiber dispersion on multiple cracking of cement composites. ASCE Journal of Engineering Mechanics. 127. (2001) 311-6.

3. J.E. Bolander, S. Choi, and S.R. Duddukuri, Fracture of fiber-reinforced cement composites: effects of fiber dispersion. International Journal of Fracture. 154. (2008) 73-86.

4. B. Shen, M. Hubler, G.H. Paulino, and L.J. Struble, Functionally-graded fiber-reinforced cement composite: processing, microstructure, and properties. Cement and Concrete Composites. 30. (2008) 663-73.

5. J. Kang, K. Kim, Y.M. Lim, and J.E. Bolander, Modeling of fiber-reinforced cement composites: discrete representation of fiber pullout. International Journal of Solids and Structures. 51. (2014) 1970-9.

6. A.E. Naaman, G.G. Namur, J.M. Alwan, and H.S. Najm, Fiber pullout and bond slip. I: analytical study. ASCE Journal of Structural Engineering. 117. (1991) 2769-90.

7. M. Kunieda, H. Ogura, N. Ueda, and H. Nakamura, Tensile fracture process of strain hardening cementitious composites by means of three-dimensional meso-scale analysis. Cement and Concrete Composites. 33. (2011) 956-65. 